\title{
UNIQUENESS OF DIFFERENCE-DIFFERENTIAL POLYNOMIALS OF ENTIRE FUNCTIONS SHARING ONE VALUE
}

\author{
RENUKADEVI S. DYAVANAL AND ASHWINI M. HATTIKAL
}

\begin{abstract}
In this paper, we study the uniqueness of difference-differential polynomials of entire functions $f$ and $g$ sharing one value with counting multiplicity. In this paper we extend and generalize the results of X. Y. Zhang, J. F. Chen and W. C. Lin [17], L. Kai, L. Xin-ling and C. Ting-bin [7] and many others [2, 16].
\end{abstract}

\section{Introduction and main results}

In this paper, the term 'meromorphic' will always mean meromorphic in the whole complex plane $\overline{\mathbb{C}}$. It is assumed that the reader is familiar with standard notations and fundamental results of Nevanlinna theory [6], [13] and [15]. We denote by $S(r, f)$ any quantity satisfying $S(r, f)=o\{T(r, f)\}$ as $r \longrightarrow+\infty$, possibly outside of a set of finite linear measure.

For $a \in \overline{\mathbb{C}}$ and $k$ be a positive integer, we denote by $N_{(k}(r, a, f)$ be the counting function for the zeros of $f(z)-a$ with multiplicity $\geq k$, and $\bar{N}_{(k}(r, a, f)$ be the corresponding one for which the multiplicity is not counted. In this paper, we denote by

$$
N_{k}(r, a, f)=\bar{N}_{(1}(r, a, f)+\bar{N}_{(2}(r, a, f)+\ldots+\bar{N}_{(k}(r, a, f)
$$

Let $f(z)$ and $g(z)$ be two meromorphic functions. If $f(z)-a$ and $g(z)-a$ assume the same zeros with the same multiplicities, then we say that $f(z)$ and $g(z)$ share the value ' $a$ ' CM, where ' $a$ ' is a complex number.

In 1993, Wang and Fang $[11,12]$ proved the following theorem for transcendental entire functions.

Theorem A. Let $f(z)$ be a transcendental entire function. $n$ and $k$ be two positive integers with $n \geq k+1$, then $\left[f^{n}\right]^{(k)}-1$ has infinitely many zeros.

Received Febuary 23, 2015, accepted August 20, 2015. 2010 Mathematics Subject Classification. 30D35, 39A05.

Key words and phrases. Nevanlinna theory, Entire functions, Difference-differential polynomials, Sharing value, Uniqueness, etc..

Corresponding author: Renukadevi S. Dyavanal. 
In 2002, M. L. Fang [3] proved the unicity theorem corresponding to the above result.

Theorem B. Let $f$ and $g$ be two non-constant entire functions, and let $n \geq 11$ be a positive integer with $n>2 k+4$. If $\left[f^{n}\right]^{(k)}$ and $\left[g^{n}\right]^{(k)}$ share $1 C M$, then either $f(z)=c_{1} e^{c z}, g(z)=c_{2} e^{-c z}$, where $c_{1}, c_{2}$ and c are three constants satisfying $(-1)^{k}\left(c_{1} c_{2}\right)^{n}(n c)^{2 k}=1$, or $f \equiv \operatorname{tg}$ for a constant $t$ such that $t^{n}=1$.

In 2008, X. Y. Zhang, J. F. Chen and W. C. Lin [17] proved the following results on uniqueness of two polynomials sharing a common value.

Theorem C. Let $f$ be a transcendental entire function, let $n, k$ and $m$ be positive integers with $n \geq k+2$, and $P(z)=a_{0}+a_{1} z+a_{2} z^{2}+\ldots+a_{m} z^{m}$, where $a_{0}, a_{1}, a_{2}, \ldots, a_{m}$ are complex constants. Then $\left[f^{n} P(f)\right]^{(k)}=1$ has infinitely many solutions.

Theorem D. Let $f$ and $g$ be two non-constant entire functions. Let $n, k$ and $m$ be three positive integers with $n \geq 3 m+2 k+5$, and $P(z)=a_{m} z^{m}+a_{m-1} z^{m-1}+\ldots+a_{1} z+a_{0}$ or $P(z) \equiv c_{0}$, where $a_{0}(\neq 0), a_{1}, a_{2}, a_{3}, \ldots a_{m-1}, a_{m}(\neq 0), c_{0}(\neq 0)$ are complex constants. If $\left[f^{n} P(f)\right]^{(k)}$ and $\left[g^{n} P(g)\right]^{(k)}$ share $1 C M$, then

(1) when $P(z)=a_{m} z^{m}+a_{m-1} z^{m-1}+\ldots+a_{1} z+a_{0}$, either $f(z) \equiv \operatorname{tg}(z)$ for a constant $t$ such that $t^{d}=1$, where $d=(n+m, \ldots . n+m-i, \ldots, n), a_{m-i}(\neq 0)$ for some $i=0,1,2, \ldots m$, or $f$ and $g$ satisfy the algebraic equation $R(f, g) \equiv 0$, where $R\left(w_{1}, w_{2}\right)=w_{1}^{n}\left(a_{m} w_{1}^{m}+a_{m-1} w_{1}^{m-1}+\right.$ $\left.\ldots+a_{0}\right)-w_{2}^{n}\left(a_{m} w_{2}^{m}+a_{m-1} w_{2}^{m-1}+\ldots+a_{0}\right)$

(2) when $P(z) \equiv c_{0}$, either $f(z)=\frac{c_{1}}{\sqrt[n]{c_{0}} e^{c z}}, g(z)=\frac{c_{2}}{\sqrt[n]{c_{0}} e^{-c z}}$, where $c_{1}, c_{2}$ and c are constants satisfying $(-1)^{k}\left(c_{1} c_{2}\right)^{n}(n c)^{2 k}=1$, or $f \equiv t g$ for a constant $t$ such that $t^{n}=1$.

In 2012, L. Kai, L. Xin-ling, C. Ting-bin [7] considered Theorem B for difference-differential polynomials and proved the following results.

Theorem E. Let $f(z)$ be a transcendental entire function of finite order. If $n \geq k+2$, then the difference-differential polynomial $\left[f^{n}(z) f(z+c)\right]^{(k)}-\alpha(z)$ has infinitely many zeros.

Theorem F. Let $f$ and $g$ be transcendental entire functions of finite order, $n \geq 2 k+6$ and $c$ is a non-zero complex constant. If $\left[f^{n}(z) f(z+c)\right]^{(k)}$ and $\left[g^{n}(z) g(z+c)\right]^{(k)}$ share the value $1 C M$, then either $f(z)=c_{1} e^{C z}, g(z)=c_{2} e^{-C z}$, where $c_{1}, c_{2}$ and C are constants satisfying $(-1)^{k}\left(c_{1} c_{2}\right)^{n+1}((n+$ 1)C $)^{2 k}=1$, or $f \equiv$ tg for a constant $t$ such that $t^{n+1}=1$.

In the same direction J. Zhang [16] investigated the value distribution and uniqueness of difference polynomials of entire functions and obtained the following results.

Theorem G. Let $f(z)$ be a transcendental entire function of finite order, and $\alpha(z)$ be a small function with respect to $f(z)$. Suppose that $c$ is a non-zero complex constant. If $n \geq 2$, then $f^{n}(z)(f(z)-1) f(z+c)-\alpha(z)$ has infinitely many zeros. 
Theorem H. Let $f$ and $g$ be two transcendental entire functions of finite order, and $\alpha(z)$ be a small function with respect to both $f(z)$ and $g(z)$. Suppose that $c$ is a non-zero constant and $n$ is an integer. If $n \geq 7$, then $f^{n}(z)(f(z)-1) f(z+c)$ and $g^{n}(z)(g(z)-1) g(z+c)$ share $\alpha(z) C M$, then $f(z) \equiv g(z)$.

Recently, R. S. Dyavanal and R. V. Desai [2] extended the results of J. Zhang[16] and proved the following results.

Theorem I. Let $f(z)$ be a transcendental entire function of finite order, and $\alpha(z)$ be a small function with respect to $f(z)$. Suppose thatc is a non-zero complex constant and $n$ is an integer. If $n \geq 2, k_{1} \geq 1$ then $f^{n}(z)(f(z)-1)^{k_{1}} f(z+c)-\alpha(z)$ has infinitely many zeros.

Theorem J. Let $f(z)$ and $g(z)$ be two transcendental entire functions of finite order, and $\alpha(z)$ be a small function with respect to both $f(z)$ and $g(z)$. Suppose that $c$ is a non-zero complex constant, $k_{1} \geq 1, n \geq k_{1}+6$. If $f^{n}(z)(f(z)-1)^{k_{1}} f(z+c)$ and $g^{n}(z)(g(z)-1)^{k_{1}} g(z+c)$ share $\alpha(z) C M$, then $f(z) \equiv \operatorname{tg}(z)$, where $t^{k_{1}}=1$.

In this paper, we consider Theorem $\mathrm{C}$ and Theorem $\mathrm{D}$ to difference-differential polynomials and extends the above theorems as follows.

Theorem 1.1. Let $f$ be a transcendental entire function. $n, k$ and $m$ be positive integers with $n \geq k+2$ and $P(z)=a_{m} z^{m}+a_{m-1} z^{m-1}+\ldots+a_{1} z+a_{0}$, where $a_{0}, a_{1}, a_{2}, a_{3}, \ldots a_{m-1}, a_{m}$ are complex constants and $\alpha(z)$ be a small function with respect to $f(z)$. Then $\left[f^{n}(z) P(f) f(z+\right.$ $c)]^{(k)}-\alpha(z)$ has infinitely many zeros.

Remark 1.1. If $P(f)=1$ in Theorem 1.1, then Theorem 1.1 reduces to Theorem E.

Remark 1.2. If $P(f)=(f-1)$ and $k=0$ in Theorem 1.1, then Theorem 1.1 reduces to Theorem G.

Remark 1.3. If $P(f)=(f-1)^{k_{1}}$ and $k=0$ in Theorem 1.1, then Theorem 1.1 reduces to Theorem I.

The unicity theorem corresponding to Theorem 1.1 is as follows.

Theorem 1.2. Let $f$ and $g$ be two non-constant entire functions of finite order. Let $n, k$ and $m$ be three positive integers with $n \geq m+2 k+6$, ' $c$ ' is a non-zero complex constant and $P(z)=$ $a_{m} z^{m}+a_{m-1} z^{m-1}+\ldots+a_{1} z+a_{0}$ or $P(z) \equiv c_{0}$, where $a_{0}(\neq 0), a_{1}, a_{2}, a_{3}, \ldots, a_{m-1}$, $a_{m}(\neq 0), c_{0}(\neq 0)$ are complex constants. If $\left[f^{n}(z) P(f) f(z+c)\right]^{(k)}$ and $\left[g^{n}(z) P(g) g(z+c)\right]^{(k)}$ share $1 C M$, then

(1) when $P(z)=a_{m} z^{m}+a_{m-1} z^{m-1}+\ldots+a_{1} z+a_{0}$, we get $f(z) \equiv \operatorname{tg}(z)$ for a constant $t$ such that $t^{d}=1$, where $d=G C D\{n+m+1, n+m, \ldots, n+m+1-i, \ldots, n+1\}$ and $i=0,1,2, \ldots, m$. 
(2) when $P(z) \equiv c_{0}$ either $f(z)=\frac{c_{1} e^{C z}}{\sqrt[n]{c_{0}}}, g(z)=\frac{c_{2} e^{-C z}}{\sqrt[n]{c_{0}}}$, where $c_{1}, c_{2}, c_{0}$ and $C$ are constants satisfying $(-1)^{k}\left(c_{1} c_{2}\right)^{n+1}((n+1) C)^{2 k}=\left(\sqrt[n]{c_{0}}\right)^{2}$, or $f \equiv \operatorname{tg}$ for a constant $t$ such that $t^{n+1}=1$.

Remark 1.4. If $P(f)=1$ in Theorem 1.2, then Theorem 1.2 reduces to Theorem $\mathrm{F}$.

Remark 1.5. If $P(f)=(f-1)$ and $k=0$ in Theorem 1.2, then Theorem 1.2 reduces to Theorem $\mathrm{H}$, when $\alpha(z)=1$.

Remark 1.6. If $P(f)=(f-1)^{k_{1}}$ and $k=0$ in Theorem 1.2, then Theorem 1.2 reduces to Theorem J, when $\alpha(z)=1$.

\section{Some lemmas}

For the proof of our main results, we need the following lemmas.

Lemma 2.1 ([1]). Let $f(z)$ be a trancendental meromorphic function of finite order, then

$$
T(r, f(z+c))=T(r, f)+S(r, f)
$$

Lemma 2.2 ([15]). Let $f(z)$ be a non-constant meromorphic function, and $a_{n}(\neq 0), a_{n-1}$, $\ldots, a_{0}$ be small functions with respect to $f$. Then

$$
T\left(r, a_{n} f^{n}+a_{n-1} f^{n-1}+\ldots+a_{1} f+a_{0}\right)=n T(r, f)+S(r, f)
$$

Lemma 2.3 ([5]). Let $f$ be a transcendental meromorphic function of finite order. Then

$$
m\left(r, \frac{f(z+c)}{f(z)}\right)=S(r, f)
$$

Lemma 2.4 ([6, 13]). Let $f(z)$ be a non-constant meromorphic function and $a_{1}(z), a_{2}(z)$ be two meromorphic functions such that $T\left(r, a_{i}\right)=S(r, f), i=1,2$. Then

$$
T(r, f) \leq \bar{N}(r, f)+\bar{N}\left(r, \frac{1}{f-a_{1}}\right)+\bar{N}\left(r, \frac{1}{f-a_{2}}\right)+S(r, f)
$$

Lemma 2.5 ([15]). Let $f(z)$ and $g(z)$ be two trancendental entire functions, and $k$ be a positive integer. Then

$$
T\left(r, f^{(k)}\right) \leq T(r, f)+k \bar{N}(r, f)+S(r, f)
$$

Lemma $2.6([1],[4])$. Let $f(z)$ be a meromorphic function of finite order and $c$ is a non-zero complex constant. Then

$$
m\left(r, \frac{f(z+c)}{f(z)}\right)+m\left(r, \frac{f(z)}{f(z+c)}\right)=S(r, f)
$$


Lemma 2.7 (Lemma 3 in [14]). Let $F$ and $G$ be non-constant meromorphic functions. If $F$ and $G$ share 1 CM, then one of the following three cases holds

(1) $\max \{T(r, F), T(r, G)\} \leq N_{2}\left(r, \frac{1}{F}\right)+N_{2}(r, F)+N_{2}\left(r, \frac{1}{G}\right)+N_{2}(r, G)+S(r, F)+S(r, G)$,

(2) $F \equiv G$

(3) $F G \equiv 1$.

Lemma 2.8 ([8], Lemma 2.3). Let $f(z)$ be a non-constant meromorphic function and $p, k$ be positive integers. Then

$$
\begin{array}{r}
N_{p}\left(r, \frac{1}{f^{(k)}}\right) \leq T\left(r, f^{(k)}\right)-T(r, f)+N_{p+k}\left(r, \frac{1}{f}\right)+S(r, f) \\
N_{p}\left(r, \frac{1}{f^{(k)}}\right) \leq k \bar{N}(r, f)+N_{p+k}\left(r, \frac{1}{f}\right)+S(r, f)
\end{array}
$$

Lemma 2.9. Let $f(z)$ be a transcendental entire function of finite order and let $F^{*}=f(z)^{n} P(f) f(z+c)$. Then

$$
T\left(r, F^{*}\right)=(n+m+1) T(r, f)+S(r, f)
$$

Proof. Since $f$ is a transcendental entire function and also from Lemma 2.2, Lemma 2.3, Lemma 2.6, we obtain

$$
\begin{aligned}
(n+m+1) T(r, f)+S(r, f) & =T\left(r, f(z)^{n+1} P(f)\right) \leq m\left(r, f(z)^{n+1} P(f)\right)+S(r, f) \\
& \leq m\left(r, \frac{f(z) F^{*}}{f(z+c)}\right)+S(r, f) \\
& \leq m\left(r, F^{*}\right)+S(r, f) \\
& \leq T\left(r, F^{*}\right)+S(r, f)
\end{aligned}
$$

On the other hand, using Lemma 2.1 and $f$ is a transcendental entire function of finite order, we have

$$
\begin{aligned}
T\left(r, F^{*}\right) & \leq n T(r, f)+m T(r, f)+T(r, f(z+c))+S(r, f) \\
& \leq(n+m+1) T(r, f)+S(r, f)
\end{aligned}
$$

Hence we get Lemma 2.9.

Lemma 2.10. Let $f(z)$ and $g(z)$ be two non-constant entire functions, let $n, k$ be two positive integers with $n>k,{ }^{\prime} c^{\prime}$ is a non-zero complex constant and let $P(z)=a_{m} z^{m}+a_{m-1} z^{m-1}+\ldots+$ $a_{1} z+a_{0}$ be a non-zero polynomial, where $a_{0}, a_{1}, a_{2}, \ldots a_{m-1}, a_{m}$ are complex constants. If $\left[f^{n} P(f) f(z+c)\right]^{(k)}\left[g^{n} P(g) g(z+c)\right]^{(k)} \equiv 1$, then $P(z)$ is reduced to a non-zero monomial, that is $P(z)=a_{i} z^{i} \neq 0$ for some $i=0,1,2, \ldots, m$. 
Proof. If $P(z)$ is not reduced to a non-zero monomial, then without loss of generality, we may assume that

$$
P(z)=a_{m} z^{m}+a_{m-1} z^{m-1}+\ldots+a_{1} z+a_{0}
$$

where $a_{0}(\neq 0), a_{1}, a_{2}, \ldots a_{m-1}, a_{m}(\neq 0)$ are complex constants. Since

$$
\left[f^{n}\left(a_{m} f^{m}+\ldots+a_{0}\right) f(z+c)\right]^{(k)}\left[g^{n}\left(a_{m} g^{m}+\ldots+a_{0}\right) g(z+c)\right]^{(k)} \equiv 1
$$

From $n>k$ and the assumption that $f(z)$ and $g(z)$ are two non-constant entire functions we deduce by (2.1) that

$$
f(z) \neq 0, g(z) \neq 0 \text {. }
$$

Let $f(z)=e^{\alpha(z)}$, where $\alpha(z)$ is a non-constant entire function. Thus, by induction we get

$$
\left[a_{i} f^{i+n} f(z+c)\right]^{(k)}=P_{i}\left(\alpha^{\prime}, \ldots \alpha^{(k)}, \alpha^{\prime}(z+c), \ldots \alpha^{(k)}(z+c)\right) e^{(i+n) \alpha} e^{\alpha(z+c)} .
$$

Where $P_{i}\left(\alpha^{\prime}, \alpha^{\prime \prime}, \ldots \alpha^{(k)}, \alpha^{\prime}(z+c), \alpha^{\prime \prime}(z+c), \ldots \alpha^{(k)}(z+c)\right)(i=0,1, \ldots m)$ are difference-differential polynomials.

Obviously,

$$
\begin{gathered}
P_{m}\left(\alpha^{\prime}, \ldots \alpha^{(k)}, \alpha^{\prime}(z+c), \ldots \alpha^{(k)}(z+c)\right) \not \equiv 0 \\
\vdots \\
P_{0}\left(\alpha^{\prime}, \ldots \alpha^{(k)}, \alpha^{\prime}(z+c), \ldots \alpha^{(k)}(z+c)\right) \not \equiv 0
\end{gathered}
$$

Where if $a_{i} \neq 0$ for some $i=1,2 \ldots m-1$, then $P_{i}\left(\alpha^{\prime} \ldots \alpha^{(k)}, \alpha^{\prime}(z+c), \ldots \alpha^{(k)}(z+c)\right) \not \equiv 0$ Since $g(z)$ is an entire function, we get from (2.1) that $\left[f^{n}\left(a_{m} f^{m}+\ldots+a_{0}\right) f(z+c)\right]^{(k)} \neq 0$. Thus, by (2.3) we have

$$
\begin{aligned}
& P_{m}\left(\alpha^{\prime}, \alpha^{\prime \prime}, \ldots \alpha^{(k)}, \alpha^{\prime}(z+c), \alpha^{\prime \prime}(z+c), \ldots \alpha^{(k)}(z+c)\right) e^{m \alpha}+\ldots \\
& +\ldots P_{0}\left(\alpha^{\prime}, \alpha^{\prime \prime}, \ldots \alpha^{(k)}, \alpha^{\prime}(z+c), \alpha^{\prime \prime}(z+c), \ldots \alpha^{(k)}(z+c)\right) \not \equiv 0
\end{aligned}
$$

Since $\alpha(z)$ and $\alpha(z+c)$ is an entire function, we obtain

$$
\begin{aligned}
T\left(r, \alpha^{(j)}\right) \leq T\left(r, \alpha^{\prime}\right)+S(r, f) & =m\left(r, \alpha^{\prime}\right)+S(r, f) \\
& =m\left(r, \frac{\left(e^{\alpha}\right)^{\prime}}{e^{\alpha}}\right)+S(r, f)
\end{aligned}
$$

Similarly, we obtain

$$
T\left(r, \alpha^{(j)}(z+c)\right) \leq T\left(r, \alpha^{\prime}(z+c)\right)+S(r, f)=m\left(r, \alpha^{\prime}(z+c)\right)+S(r, f)
$$




$$
=m\left(r, \frac{\left(e^{\alpha(z+c)}\right)^{\prime}}{e^{\alpha(z+c)}}\right)+S(r, f)
$$

for $j=1,2, \ldots, k$. Hence, we deduce that

$$
T\left(r, P_{m}\right)=S(r, f), \ldots, T\left(r, P_{0}\right)=S(r, f)
$$

Note that $f=e^{\alpha(z)}$. Thus, by (2.4), (2.5) above, and Lemma 2.2 and Lemma 2.4, we get

$$
\begin{aligned}
m T(r, f) & =T\left(r, P_{m} e^{m \alpha}+\ldots+P_{1} e^{\alpha}\right)+S(r, f) \\
& \leq \bar{N}\left(r, \frac{1}{P_{m} e^{m \alpha}+\ldots+P_{1} e^{\alpha}}\right)+\bar{N}\left(r, \frac{1}{P_{m} e^{m \alpha}+\ldots+P_{1} e^{\alpha}+P_{0}}\right)+S(r, f) \\
& \leq \bar{N}\left(r, \frac{1}{P_{m} e^{(m-1) \alpha}+\ldots+P_{2} e^{\alpha}+P_{1}}\right)+S(r, f) \\
& \leq(m-1) T(r, f)+S(r, f)
\end{aligned}
$$

which is a contradiction. This shows that $P(z)$ is reduced to a non-zero monomial, that is, $P(z)=a_{i} z^{i} \neq 0$ for some $i=0,1,2, \ldots m$. This completes the proof of the Lemma 2.10.

\section{Proof of Theorem 1.1}

Denote $F(z)=\left[f(z)^{n} P(f) f(z+c)\right]^{(k)}$ and $F^{*}=f(z)^{n} P(f) f(z+c)$. From Lemma 2.9, $F^{*}$ is not a constant. Assume that $F(z)-\alpha(z)$ has only finitely many zeros, then from the second fundamental theorem for three values and (1) of Lemma 2.8, we get

$$
\begin{aligned}
T(r, F) & \leq \bar{N}(r, F)+\bar{N}(r, 0, F)+\bar{N}(r, 0, F-\alpha(z))+S(r, F) \\
& \leq N_{1}(r, 0, F)+\bar{N}(r, 0, F-\alpha(z))+S(r, F) \\
& \leq T(r, F)-T\left(r, F^{*}\right)+N_{k+1}\left(r, 0, F^{*}\right)+S\left(r, F^{*}\right)+S(r, F) \\
T\left(r, F^{*}\right) & \leq N_{k+1}\left(r, 0, F^{*}\right)+S(r, f) .
\end{aligned}
$$

From Lemma 2.9 and (3.1), it implies that

$$
\begin{aligned}
(n+m+1) T(r, f)+S(r, f) & =T\left(r, F^{*}\right) \leq N_{k+1}\left(r, 0, F^{*}\right)+S(r, f) \\
& \leq(k+1) \bar{N}(r, 0, f)+N(r, 0, P(f))+N(r, 0, f(z+c))+S(r, f) \\
& \leq(k+m+2) T(r, f)+S(r, f) .
\end{aligned}
$$

Which is contradiction to $n \geq k+2$. Hence $\left[f^{n}(z)\left(a_{m} f^{m}+\ldots+a_{0}\right) f(z+c)\right]^{(k)}-\alpha(z)$ has infinitely many zeros.

Proof of theorem 1.2.

(1) If $P(z)=a_{m} z^{m}+a_{m-1} z^{m-1}+\ldots+a_{1} z+a_{0}$. 
Then by assumption and Theorem 1.1 we know that either both $f$ and $g$ are transcendental entire functions or both $f$ and $g$ are polynomials.

First, we consider the case when $f$ and $g$ are transcendental entire functions.

Considering $F=\left[f^{n}(z) P(f) f(z+c)\right]^{(k)}, G=\left[g^{n}(z) P(g) g(z+c)\right]^{(k)}$. Since $F$ and $G$ share $1 \mathrm{CM}$, let us assume (1) of Lemma 2.7 holds. That is

$$
\max \{T(r, F), T(r, G)\} \leq N_{2}\left(r, \frac{1}{F}\right)+N_{2}(r, F)+N_{2}\left(r, \frac{1}{G}\right)+N_{2}(r, G)+S(r, F)+S(r, G)
$$

Since $f$ is an entire function and from Lemma 2.5, Lemma 2.9 we have $S(r, F)=S(r, f)$. We also have $S(r, G)=S(r, g)$.

From (1) of Lemma 2.8, we obtain

$$
\begin{aligned}
N_{2}\left(r, \frac{1}{F}\right) & =N_{2}\left(r, \frac{1}{\left[f^{n}(z) P(f) f(z+c)\right]}{ }^{(k)}\right) \\
& \leq T(r, F)-T\left(r, f^{n}(z) P(f) f(z+c)\right)+N_{k+2}\left(r, \frac{1}{f^{n}(z) P(f) f(z+c)}\right)+S(r, f) .
\end{aligned}
$$

From Lemma 2.9 and (3.3), we get

$$
\begin{aligned}
(n+m+1) T(r, f) & =T\left(r, f^{n}(z) P(f) f(z+c)\right)+S(r, f) \\
& \leq T(r, F)-N_{2}\left(r, \frac{1}{F}\right)+N_{k+2}\left(r, \frac{1}{f^{n}(z) P(f) f(z+c)}\right)+S(r, f)
\end{aligned}
$$

From (2) of Lemma 2.8, we get

$$
\begin{aligned}
N_{2}\left(r, \frac{1}{F}\right) & \leq N_{k+2}\left(r, \frac{1}{f^{n}(z) P(f) f(z+c)}\right) \\
& \leq(k+2) N\left(r, \frac{1}{f}\right)+m N\left(r, \frac{1}{f}\right)+N\left(r, \frac{1}{f(z+c)}\right) \\
& \leq(k+m+3) T(r, f)+S(r, f) .
\end{aligned}
$$

Similarly as above, we obtain

$$
\begin{aligned}
(n+m+1) T(r, g) & \leq T(r, G)-N_{2}\left(r, \frac{1}{G}\right)+N_{k+2}\left(r, \frac{1}{g^{n}(z) P(g) g(z+c)}\right)+S(r, g) \\
N_{2}\left(r, \frac{1}{G}\right) & \leq N_{k+2}\left(r, \frac{1}{g^{n}(z) P(g) g(z+c)}\right) \leq(k+m+3) T(r, g)+S(r, g) .
\end{aligned}
$$

Using equations (3.2)-(3.7), we deduce

$$
\begin{aligned}
(n+m+1)[T(r, f)+T(r, g)] \leq & 2 N_{k+2}\left(r, \frac{1}{f(z)^{n} P(f) f(z+c)}\right)+2 N_{k+2}\left(r, \frac{1}{g(z)^{n} P(g) g(z+c)}\right) \\
& +S(r, f)+S(r, g) \\
\leq & 2(k+m+3)[T(r, f)+T(r, g)]+S(r, f)+S(r, g) .
\end{aligned}
$$


Which is contradiction to $n \geq m+2 k+6$. Hence, by Lemma 2.7, we get either $F G \equiv 1$ or $F \equiv G$. Suppose $F G \equiv 1$ holds,

$$
\text { i.e., }\left[f^{n}(z)\left(a_{m} f^{m}+\ldots+a_{0}\right) f(z+c)\right]^{(k)}\left[g^{n}(z)\left(a_{m} g^{m}+\ldots+a_{0}\right) g(z+c)\right]^{(k)} \equiv 1
$$

By assumption that $a_{m} \neq 0, a_{0} \neq 0$, we can arrive at a contradiction by Lemma 2.10 . Hence, by Lemma $2.7 F(z) \equiv G(z)$,

$$
\text { i.e., }\left[f^{n}(z)\left(a_{m} f^{m}+\ldots+a_{0}\right) f(z+c)\right]^{(k)} \equiv\left[g^{n}(z)\left(a_{m} g^{m}+\ldots+a_{0}\right) g(z+c)\right]^{(k)}
$$

From (3.9), we get

$$
f^{n}(z) P(f) f(z+c) \equiv g^{n}(z) P(g) g(z+c)+q(z)
$$

where $q(z)$ is a polynomial of degree at most $k-1$. If $q(z) \not \equiv 0$, then we have

$$
\frac{f^{n}(z) P(f) f(z+c)}{q(z)}=\frac{g^{n}(z) P(g) g(z+c)}{q(z)}+1
$$

Thus, from the second main theorem for three small values and (3.10), we have

$$
\begin{aligned}
(n+m+1) T(r, f) \leq & T\left(r, \frac{f^{n}(z) P(f) f(z+c)}{q(z)}\right)+S(r, f) \\
\leq & \bar{N}\left(r, \frac{f^{n}(z) P(f) f(z+c)}{q(z)}\right)+\bar{N}\left(r, \frac{q(z)}{f^{n}(z) P(f) f(z+c)}\right) \\
& +\bar{N}\left(r, \frac{q(z)}{g^{n}(z) P(g) g(z+c)}\right)+S(r, f) \\
\leq & \bar{N}\left(r, \frac{1}{f(z)}\right)+\bar{N}\left(r, \frac{1}{P(f)}\right)+\bar{N}\left(r, \frac{1}{f(z+c)}\right)+\bar{N}\left(r, \frac{1}{g(z)}\right)+\bar{N}\left(r, \frac{1}{P(g)}\right) \\
& +\bar{N}\left(r, \frac{1}{g(z+c)}\right)+S(r, f) \\
\leq & (2+m) T(r, f)+(2+m) T(r, g)+S(r, f) .
\end{aligned}
$$

Similarly as above, we have

$$
(n+m+1) T(r, g) \leq(2+m) T(r, g)+(2+m) T(r, f)+S(r, g)
$$

Thus, we get

$$
(n+m+1)[T(r, f)+T(r, g)] \leq 2(2+m)([T(r, f)+T(r, g)]+S(r, f)+S(r, g)
$$

which is contradiction to $n \geq m+2 k+6$. Hence, we get $q(z) \equiv 0$, which implies that

$$
\begin{gathered}
f^{n}(z) P(f) f(z+c)=g^{n}(z) P(g) g(z+c) \\
\text { i.e., } \quad f^{n}(z)\left(a_{m} f^{m}+a_{m-1} f^{m-1}+\ldots+a_{0}\right) f(z+c)
\end{gathered}
$$




$$
\equiv g^{n}(z)\left(a_{m} g^{m}+a_{m-1} g^{m-1}+\ldots+a_{0}\right) g(z+c) .
$$

Let $h=\frac{f}{g}$, and then substituting $f=g h$ and $f(z+c)=g(z+c) h(z+c)$ in (3.11) we deduce

$$
\begin{aligned}
& \Rightarrow(g h)^{n}\left[a_{m}(g h)^{m}+a_{m-1}(g h)^{m-1}+\ldots+a_{0}\right] g(z+c) h(z+c)=g^{n}\left(a_{m} g^{m}+\ldots+a_{0}\right) g(z+c) \\
& \Rightarrow g^{n+m}\left[a_{m}\left(h^{m+n}(z) h(z+c)-1\right)\right]+\ldots+g^{n}\left[a_{0}\left(h^{n}(z) h(z+c)-1\right)\right]=0 \\
& \Rightarrow g^{m}=\frac{-\left[g^{m-1}\left[a_{m-1}\left(h^{m+n-1}(z) h(z+c)-1\right)\right]+\ldots+\left[a_{0}\left(h^{n}(z) h(z+c)-1\right)\right]\right]}{a_{m}\left(h^{m+n}(z) h(z+c)-1\right)}
\end{aligned}
$$

If $h^{n+m}(z) h(z+c) \neq 1$, then since $g$ is transcendental and from (3.12), we have $h(z)$ is a transcendental meromorphic function with finite order. By Lemma 2.1,

$$
T(r, h(z+c))=T(r, h)+S(r, h)
$$

From (3.13) and using the condition $n \geq m+2 k+6$, it is easy to show that $h^{n+m}(z) h(z+c)$ is not a constant.

Suppose that there exist a point $z_{0}$ such that $h^{n+m}\left(z_{0}\right) h\left(z_{0}+c\right)=1$.

Since $g(z)$ is an entire function and from (3.12), we deduce $h^{d}\left(z_{0}\right)=1$, where $d=G C D\{n+$ $m+1, n+m, \ldots, n+m+1-i, \ldots, n+1\}$ and $i=0,1,2, \ldots, m$.

Now denote $H=h^{n+m}(z) h(z+c)$, then

$$
\bar{N}\left(r, \frac{1}{H-1}\right) \leq \bar{N}\left(r, \frac{1}{h^{d}(z)-1}\right) \leq d T(r, h)+O(1) \leq m T(r, h)+O(1)
$$

Applying the second fundamental theorem to $H$, and using (3.13) and (3.14), we have

$$
\begin{aligned}
T(r, H) & \leq \bar{N}(r, H)+\bar{N}\left(r, \frac{1}{H}\right)+\bar{N}\left(r, \frac{1}{H-1}\right)+S(r, h) \\
& \leq \bar{N}(r, H)+\bar{N}\left(r, \frac{1}{H}\right)+m T(r, h)+S(r, h) \\
& \leq(4+m) T(r, h)+S(r, h)
\end{aligned}
$$

Noting this, we have

$$
\begin{aligned}
(n+m) T(r, h) & =T\left(r, h^{n+m}(z)\right)=T\left(r, \frac{H}{h(z+c)}\right) \\
& \leq T(r, H)+T(r, h(z+c))+O(1) \\
& \leq(4+m) T(r, h)+T(r, h)+S(r, h) \\
& =(5+m) T(r, h)+S(r, h)
\end{aligned}
$$

which is contradiction to $n \geq m+2 k+6$.

Hence $H=h^{n+m}(z) h(z+c) \neq 1$, then 1 is picard's exceptional value of $H$, then by Second fundamental theorem, we have

$$
T(r, H) \leq \bar{N}(r, H)+\bar{N}\left(r, \frac{1}{H}\right)+\bar{N}\left(r, \frac{1}{H-1}\right)+S(r, H)
$$




$$
\begin{aligned}
& \leq 2 T(r, h)+2 T(r, h)+S(r, h) \\
(n+m+1) T(r, h) & \leq 4 T(r, h)+S(r, h)
\end{aligned}
$$

Which is contradiction to $n \geq m+2 k+6$.

$\therefore h^{n+m}(z) h(z+c) \equiv 1$, then, from (3.12), we get $h^{n}(z) h(z+c) \equiv 1 \Rightarrow h^{d}(z) \equiv 1$.

Hence, we get $f(z) \equiv \operatorname{tg}(z)$, such that $t^{d}=1$, where $d=G C D\{n+m+1, n+m, \ldots, n+m+1-$ $i, \ldots, n+1\}$ and $i=0,1,2, \ldots, m$.

Now we consider the case when $f$ and $g$ are two polynomials.

By $\left[f^{n}(z) P(f) f(z+c)\right]^{(k)}$ and $\left[g^{n}(z) P(g) g(z+c)\right]^{(k)}$ share $1 \mathrm{CM}$, we have

$$
\left[f^{n}(z)\left(a_{m} f^{m}+\ldots+a_{0}\right) f(z+c)\right]^{(k)}-1=\beta\left[\left[g^{n}(z)\left(a_{m} g^{m}+\ldots+a_{0}\right) g(z+c)\right]^{(k)}-1\right]
$$

where $\beta$ is a non-zero constant. Let $\operatorname{deg} f=l$, then by (3.15) we know that $\operatorname{deg} g=l$. Differentiating the two sides of (3.15), we get

$$
f^{n-k-1}(z) q_{1}(z)=g^{n-k-1}(z) q_{2}(z)
$$

where $q_{1}(z), q_{2}(z)$ are two polynomials with $\operatorname{deg} q_{1}(z)=\operatorname{deg} q_{2}(z)=(m+k+2) l-(k+1)$. By $n \geq m+2 k+6$, we get $\operatorname{deg} f^{n-k-1}(z)=(n-k-1) l>\operatorname{deg} q_{2}(z)$.

Thus, by (3.16) we know that there exists $z_{0}$ such that $f\left(z_{0}\right)=g\left(z_{0}\right)=0$.

Hence, by (3.15) and $f\left(z_{0}\right)=g\left(z_{0}\right)=0$, we deduce that $\beta=1$, that is,

$$
\left[f^{n}(z)\left(a_{m} f^{m}+\ldots+a_{0}\right) f(z+c)\right]^{(k)}=\left[g^{n}(z)\left(a_{m} g^{m}+\ldots+a_{0}\right) g(z+c)\right]^{(k)}
$$

Thus, we have

$$
f^{n}(z)\left(a_{m} f^{m}+\ldots+a_{0}\right) f(z+c)-g^{n}(z)\left(a_{m} g^{m}+\ldots+a_{0}\right) g(z+c)=Q(z)
$$

where $Q(z)$ is a polynomial of degree atmost $k-1$. Next we prove $Q(z) \equiv 0$. By rewriting (3.17) as

$$
f^{n-k}(z) p_{1}(z)=g^{n-k}(z) p_{2}(z) .
$$

Where $p_{1}(z), p_{2}(z)$ are two polynomials with $\operatorname{deg} p_{1}(z)=\operatorname{deg} p_{2}(z)=(m+k+1) l-k$ and $\operatorname{deg} f(z)=l$.

Hence total number of common zeros of $f^{n-k}(z)$ and $g^{n-k}(z)$ is atleast $k$. Thus, by (3.18) we deduce that $Q(z) \equiv 0$, that is

$$
f^{n}(z)\left(a_{m} f^{m}+a_{m-1} f^{m-1}+\ldots+a_{0}\right) f(z+c)=g^{n}(z)\left(a_{m} g^{m}+a_{m-1} g^{m-1}+\ldots+a_{0}\right) g(z+c) .
$$

Next, similar to the argument of (3.11), we get $f(z) \equiv \operatorname{tg}(z)$ for a constant $t$ such that $t^{d}=1$, where $d=G C D\{n+m+1, n+m, \ldots, n+m+1-i, \ldots, n+1\}$ and $i=0,1,2, \ldots, m$. 
Hence proved the (1) of Theorem 1.2.

(2) If $P(z) \equiv c_{0}$

By the assumption and Theorem 1.1, we know that either both $f$ and $g$ are transcendental entire functions or both $f$ and $g$ are polynomials.

First, we consider the case when both $f$ and $g$ are transcendental entire functions. Let

$$
F=f^{n}(z) c_{0} f(z+c), G=g^{n}(z) c_{0} g(z+c)
$$

By the Theorem F and $n \geq m+2 k+6$, we obtain either $f(z)=\frac{c_{1} e^{C z}}{\sqrt[n]{c_{0}}}, g(z)=\frac{c_{2} e^{-C z}}{\sqrt[n]{c_{0}}}$, where $c_{1}, c_{2}, c_{0}$ and $C$ are constants satisfying $(-1)^{k}\left(c_{1} c_{2}\right)^{n+1}((n+1) C)^{2 k}=\left(\sqrt[n]{c_{0}}\right)^{2}$, or $f \equiv \operatorname{tg}$ for a constant $t$ such that $t^{n+1}=1$.

Now we consider the case when both $f$ and $g$ are two polynomials.

By $\left[f^{n}(z) c_{0} f(z+c)\right]^{(k)}$ and $\left[g^{n}(z) c_{0} g(z+c)\right]^{(k)}$ share $1 \mathrm{CM}$, we have

$$
\left[f^{n}(z) c_{0} f(z+c)\right]^{(k)}-1=\gamma\left[\left[g^{n}(z) c_{0} g(z+c)\right]^{(k)}-1\right] .
$$

Where $\gamma$ is a non-zero constant. Let $\operatorname{deg} f(z)=l$, then by (3.21) we know that $\operatorname{deg} g(z)=l$. Differentiating the two sides of (3.21), we get

$$
f^{n-k-1}(z) q_{3}(z)=g^{n-k-1}(z) q_{4}(z)
$$

where $q_{3}(z), q_{4}(z)$ are two polynomials with $\operatorname{deg} q_{3}(z)=\operatorname{deg} q_{4}(z)=(k+2) l-(k+1)$. By $n \geq 2 k+6$, we get $\operatorname{deg} f^{n-k-1}(z)=(n-k-1) l>\operatorname{deg} q_{4}(z)$.

Thus, by (3.22) we know that there exists $z_{0}$ such that $f\left(z_{0}\right)=g\left(z_{0}\right)=0$.

Hence, by (3.21) and $f\left(z_{0}\right)=g\left(z_{0}\right)=0$, we deduce that $\gamma=1$, that is,

$$
\left[f^{n}(z) c_{0} f(z+c)\right]^{(k)}=\left[g^{n}(z) c_{0} g(z+c)\right]^{(k)}
$$

Thus, we have

$$
f^{n}(z) f(z+c)-g^{n}(z) g(z+c)=Q_{1}(z)
$$

where $Q_{1}(z)$ is a polynomial of degree atmost $k-1$. Next we prove $Q_{1}(z) \equiv 0$. By rewriting (3.23) as

$$
f^{n-k}(z) p_{3}(z)=g^{n-k}(z) p_{4}(z)
$$

where $p_{3}(z), p_{4}(z)$ are two polynomials with $\operatorname{deg} p_{3}(z)=\operatorname{deg} p_{4}(z)=(k+1) l-k$ and $\operatorname{deg} f(z)=$ $l$.

Hence total number of common zeros of $f^{n-k}(z)$ and $g^{n-k}(z)$ is atleast $k$.

Thus, by (3.24) we deduce that $Q_{1}(z) \equiv 0$, that is,

$$
f^{n}(z) f(z+c)=g^{n}(z) g(z+c) .
$$


Let $h(z)=\frac{f(z)}{g(z)}$ and $h(z+c)=\frac{f(z+c)}{g(z+c)}$ then

$$
(g h)^{n} g(z+c) h(z+c)=g^{n} g(z+c)
$$

Hence $f=\operatorname{tg}$ where $(\operatorname{tg})^{n} \operatorname{tg}(z+c)=g^{n} g(z+c)$

$$
\Rightarrow t^{n+1}=1
$$

Hence proved the (2) of Theorem 1.2.

\section{Open questions}

Question 4.1. Can 1 point shared value in the Theorem 1.2 be replaced by fixed point?

Question 4.2. Can 1 point shared value with CM in the Theorem 1.2 be replaced by 1 point shared value with IM?

Question 4.3. Do the Theorem 1.1 and Theorem 1.2 hold for meromorphic functions $f$ and $g$ ?

Question 4.4. What happens if the CM sharing is replaced by weighted sharing of small function in Theorem 1.2?

Question 4.5. Are the conditions $n \geq k+2$ in Theorem 1.1 and $n \geq m+2 k+6$ in Theorem 1.2 sharp?

\section{Acknowledgement}

Second author was supported by UGC's Research Fellowship in Science for meritorious Students, UGC, New Delhi. Ref. No.F.7-101/2007(BSR).

\section{References}

[1] Y. M. Chiang and S. J. Feng, On the Nevanlinna characteristic of $f(z+\eta)$ and difference equations in the complex plane, Ramanujan J., 16 (2008), 105-129.

[2] R. S. Dyavanal and R. V. Desai, Uniqueness of difference polynomials of entire functions, Appl. Math. Sci., 8 (2014), 3419-3424.

[3] M. L. Fang, Uniqueness and value-sharing of entire function, Comput. Math. Appl., 44(2002), 823-831.

[4] R. G. Halburd and R. J. Korhonen, Nevanlinna theory for the difference operator, Ann. Acad. Sci. Fenn. Math., 31 (2006), 463-478.

[5] R. G. Halburd and R. J. Korhonen, Meromorphic solutions of difference equations, integrability and the discrete Painleve equations, J. Phys. A.: Math. Theor., 40 (2007), 1-38.

[6] W. K. Hayman, Meromorphic Functions, Claredon Press, Oxford, 1964.

[7] L. Kai, L. Xin-ling and C. Ting-bin, Some results on zeros and uniqueness of difference-differential polynomials, Appl. Math. J. Chinese Univ. 27 (2012), 94-104. 
[8] I. Lahiri and A. Sarkar, Uniqueness of a meromorphic function and its derivative, J. Inequal. Pure Appl. Math., 5 (2004), No. 1, Article 20, 20 Pages.

[9] I. Laine and C. C. Yang, Value distribution of difference polynomials, Proc. Japan Acad. Ser. A, 83 (2007), 148151.

[10] K. Liu and L. Z. Yang, Value distribution of the difference operator, Arch. Math., 92 (2009), 270-278.

[11] Y. F. Wang, On Mues conjecture and Picard values, Sci. China, 36 (1993), 28-35.

[12] Y. F. Wang, M. L. Fang, Picard values and normal families of meromorphic functions with multiple zeros, Acta Math Sinica, 14 (1998), 17-26.

[13] L. Yang, Value Distribution Theory, Springer-Verlag Berlin, 1993.

[14] C. C. Yang and X. H. Hua, Uniqueness and value sharing of meromorphic functions, Ann. Acad. Sci. Fenn. Math., 22 (1997), 395-406.

[15] C. C. Yang and H. X. Yi, Uniqueness Theory of Meromorphic Functions, Kluwer Academic Publishers, Dordrecht, 2003; Chinese original: Science Press, Beijing, 1995.

[16] J. L. Zhang, Value distribution and shared sets of differences of meromorphic functions, J. Math. Anal. Appl., 367 (2010), 401-408.

[17] X. Y. Zhang, J. F. Chen and W. C. Lin, Entire or meromorphic functions sharing one value, Comp. Math. Appl., 56(2008), 1876-1883.

Department of Mathematics, Karnatak University, Dharwad - 580003, India.

E-mail: renukadyavanal@gmail.com

Department of Mathematics, Karnatak University, Dharwad - 580003, India.

E-mail: ashwinimhmaths@gmail.com 